\title{
CONVERGENCE IN DISTRIBUTION OF PRODUCTS OF RANDOM MATRICES: A SEMIGROUP APPROACH
}

\author{
ARUNAVA MUKHERJEA
}

\begin{abstract}
The problem of weak convergence of the sequence of convolution powers of a probability measure has been considered in this paper in the general context of a noncompact semigroup and in particular, in the semigroup of nonnegative and real matrices. Semigroup methods have been used to give simple proofs of some recent results of Kesten and Spitzer in nonnegative matrices. It has been also shown that these methods often lead to similar results in the more general context of real matrices.
\end{abstract}

1. Introduction. Random matrices have recently attracted the attention of many probabilists. Recent results in $[\mathbf{K S}]$ are essentially concerned with the problem of weak convergence of the convolution iterates of a probability measure on a locally compact semigroup of $d \times d$ nonnegative matrices (under multiplication and usual topology) to a probability measure different from the unit mass at the zero matrix. We show here how some of the main results in $[\mathbf{K S}]$ can be obtained using certain semigroup ideas. Our methods here result in more general results for matrices with arbitrary real entries. This is the main motivation for this paper, since, as Kesten and Spitzer mention in [KS, p. 364, line 3], their methods do not apply to the case of real matrices.

2. Preliminaries. Completely simple semigroups play an important role in this paper. A semigroup is completely simple if it is simple and has a primitive idempotent. A locally compact completely simple topological semigroup $K$ is topologically isomorphic (see [MT]) to the product structure $X \times G \times Y$, where $X=E(K e)$, $G=e K e$ and $Y=E(e K)$. Here $e=e^{2}$ is a fixed idempotent element of $K$ and $E(A)$ is the set of all idempotents in $A . X, G$ and $Y$ are given relative topologies from $K$ and the multiplication in $X \times G \times Y$ is defined by

$$
(x, g, y)\left(x^{\prime}, g^{\prime}, y^{\prime}\right)=\left(x, g y x^{\prime} g^{\prime}, y^{\prime}\right) .
$$

The set $X \times G \times Y$ is given the product topology. It is well known that a compact semigroup has a closed kernel (that is, a minimum 2-sided ideal), which is completely simple. Mukherjea and Tserpes (see $[\mathbf{M T}]$ ) proved that the support of an idempotent probability measure on a locally compact (second countable Hausdorff) semigroup is a closed completely simple subsemigroup with a compact group factor. It is also known [C] that if a semigroup $S$ (of $d \times d$ matrices over a field) has a completely simple kernel $K$, then $K=m(S)$, where $m(S)$ is the set of all those

Received by the editors May 19, 1986 and, in revised form, October 28, 1986.

1980 Mathematics Subject Classification (1985 Revision). Primary 60B10, 60J10.

Key words and phrases. Nonnegative matrices, convolution of probability measures, completely simple semigroups, kernel of a semigroup. 
matrices in $S$ with the minimal rank. Another result that will play an important role here is Theorem 4.3 in $[\mathbf{M}$, p. 189]. In this theorem, the author gave a necessary and sufficient condition for the weak convergence of a tight sequence $\left(P^{n}\right)$ of the iterates of a probability measure on a noncompact semigroup $S$. It is therefore relevant to understand when $\left(P^{n}\right)$ is tight in a general noncompact semigroup. In this context, let us state the following (rather important) theorem without proof.

THEOREM 2.1. Let $S$ be a second countable locally compact Hausdorff topological semigroup and $P$ be a probability measure on the Borel sets of $S$ such that $S=\operatorname{cl}\left(\bigcup_{n=1} S_{P}^{n}\right)$. Then the convolution sequence $\left(P^{n}\right)$ is tight iff $S$ has a completely simple kernel $K$ with a compact group factor such that for every open set $G$ containing $K, \lim _{n \rightarrow \infty} P^{n}(G)=1$.

3. The convergence problem in nonnegative matrices. Here $J$ will denote the set of all $d \times d$ nonnegative matrices which have at least one zero row or one zero column or both. $J^{c}$ is the complement of $J$. Our first theorem is the simplest and perhaps the most important result in this paper. Though this result does not appear in [KS], it is influenced by the results in [KS].

THEOREM 3.1. Let $S$ be the closed semigroup generated by the support $S_{P}$ of a probability measure on $d \times d$ nonnegative matrices. Then $P_{n} \equiv(1 / n) \sum_{k=1}^{\infty} P^{k}$ converges weakly to $Q$ in $P(S)$ and $S_{Q} \cap J^{c}$ is nonempty iff $S$ is compact and $m(S) \cap J^{c}$ is nonempty.

Proof. Suppose that $P_{n}$ converges weakly to $Q$. Then $Q=Q^{2}$ and $S_{Q}$ is the completely simple kernel of $S$. Let $x \in S_{Q} \cap J^{c}$. Then $x S_{Q} x$ is a compact group and $x S_{Q} x \subset J^{c}$. Let $e=e^{2} \in x S_{Q} x$. Then $e S e=e S_{Q} e$ is a compact group of nonnegative matrices and therefore, finite. Since $e \in J^{c}, e$ has a strictly positive diagonal (see $\left[\mathbf{M}_{1}\right]$ ). Since $e S e$ is finite, there is a positive number $s$ such that for any $y \in S$ and for any $i, j(1 \leq i, j \leq d), e_{i i} y_{i j} e_{j j} \leq s$ so that $y_{i j} \leq s /\left(e_{i i} e_{j j}\right)$. This means that $S$ is compact. Since $m(S)$ is an ideal, $S_{Q} \subset m(S)$. This proves the "only if" part. The "if" part is immediate from Theorem 4.3 in [M].

Let us remark that if $S$ contains the zero matrix, then the sequence $\left(P^{n}\right)$ converges weakly to $\delta_{\{0\}}$ iff $\left(P^{n}\right)$ is tight. This is immediate from Theorem 4.3 in [M]. The next theorem covers a substantial part of Theorem 1 and Theorem 2 in [KS]. We give a different proof using semigroups.

THEOREM 3.2. Let $P$ be a (regular) probability measure on $d \times d$ nonnegative matrices such that the following two conditions hold:

$\left(K S_{1}\right) P(J)=0$

$\left(K S_{2}\right) P^{m}(I)>0$ for some positive integer $m$, where $I$ is the set of all strictly positive $d \times d$ matrices.

Let $S$ be the closed semigroup (under multiplication and usual topology) generated by $S_{P}$. Then the following statements are equivalent:

(A) $P^{n} \rightarrow Q \in P(S)$ weakly and $S_{Q} \cap J^{c} \neq \varnothing$.

(B) For each $u>0$, there exists $k_{u}>0$ such that for each positive integer $n$, $P^{n}\left(B_{u}\right)>1-u$, where $B_{u}=\left\{B \in S: \min _{i, j} B_{i, j} \leq k_{u}\right\}$ and there exists $v>0$ and a subsequence $\left(n_{t}\right)$ such that for $t \geq 1, P^{n_{t}}\left(A_{v}\right)>v$, where $A_{v}=\{B \in$ $\left.S: \max _{i, j} B_{i j}>v\right\}$.

(C) $\left(P^{n}\right)$ is tight and $P^{n} \nrightarrow \delta_{\{0\}}$ weakly. 
(D) Let $M_{n}=A_{1} A_{2} \cdots A_{n}$, where $\left(A_{i}\right)$ is an iid sequence in $S$ with common distribution $P$. Then there exists $w>0$ such that

$$
\operatorname{Pr}\left(\left\{\left(M_{n}\right)_{i j} \leq w \text { for all } i, j, \text { all } n \geq 1\right\}\right)=1
$$

and also, the second condition in (B) holds.

Proof. $(\mathrm{A}) \Rightarrow(\mathrm{B})$ Trivial.

$(\mathrm{B}) \Rightarrow(\mathrm{C})$ Assume (B). Write $I_{k}=\left\{B \in S: B_{i j} \geq 1 / k\right.$ for all $\left.i, j\right\}$. Since $I$ is an ideal of $J^{c}, P^{n}(I)$ is nondecreasing with $n$ by $\left(K S_{1}\right)$ and by a simple argument given on p. 38 on [MT], using $\left(K S_{2}\right)$,

$$
\lim _{n \rightarrow \infty} P^{n}(I)=1 \text {. }
$$

Thus there exists a positive integer $N$ and $k_{0} \geq 1$ such that

$$
P^{N}\left(I_{k_{0}}\right)>1 / 2 \text {. }
$$

Let $0<u^{\prime}<1$ and $u=u^{\prime} / 4$. Then for any positive integer $s$,

$$
\begin{aligned}
\left(1-u^{\prime}\right) / 4 & \leq \operatorname{Pr}\left(M_{2 N+s} \in B_{u}, M_{N} \in I_{k_{0}}, A_{N+s+1} \cdots A_{2 N+s} \in I_{k_{0}}\right) \\
& \leq \frac{1}{4} \cdot P^{s}\left(W_{k_{0}, u^{\prime}}\right),
\end{aligned}
$$

where $W_{k_{0}, u^{\prime}}=\left\{B: \max _{i, j} B_{i j} \leq k_{o}^{2} k_{u}\right\}$. This establishes (C).

$(\mathrm{C}) \Rightarrow(\mathrm{D})$ Immediate from Theorem 3.1 and Theorem $4.3[\mathbf{M}]$.

(D) $\Rightarrow$ (C) Trivial.

$(\mathrm{C}) \Rightarrow(\mathrm{A})$ Assume (C). By Theorem $4.3[\mathbf{M}],(1 / n) \sum_{k=1}^{n} P^{k} \rightarrow Q \in P(S)$ and $S_{Q}$ is the completely simple kernel of $S$ with a compact group factor. Clearly $0 \notin S$ by (C). Since $I \supset I \cdot S_{Q} \cdot I$ and for each positive integer $n, P^{n} * Q * P^{n}=Q$, $Q(I) \geq P^{n}(I) Q\left(S_{Q}\right) P^{n}(I)$ for each $n$. By (3.1), $Q(I)=1$. Let $x \in S_{Q} \cap I$. Then $x S_{Q} x \subset I$. But $x S_{Q} x$ is a compact group and therefore, it must be a singleton. By Theorem $4.3[\mathbf{M}],(\mathrm{A})$ follows.

We remark that under conditions of Theorem 3.2 , we can also show easily that the following are equivalent: (i) $\left(P^{n}\right)$ is tight and $0 \notin S$. (ii) $P^{n}$ converges weakly to $Q \in P(S)$ and $Q(I \cap S)=1$. (iii) $S$ is compact and $0 \notin S$. (iv) $m(S)$ is bounded and $m(S) \neq\{0\}$. Note that boundedness of $m(S)$ implies the boundedness of its closure. Then $\operatorname{cl}(m(S))$ is compact and has a kernel, which must equal $m(\operatorname{cl}(m(S)))$ by $[\mathbf{C}]$. Hence, $m(S)$ is closed. If $m(S) \neq\{0\}, 0 \notin S$. Then, $I \cap m(S) \supset(I \cap S) m(S)(I \cap S)$. As in Theorem 3.1, it follows that $S$ is compact.

In the rest of this section, we give a semigroup proof and extension of Theorem 3 [KS, p. 367]. This theorem is the most interesting (and perhaps, the most difficult) theorem in [KS]. It is stated in [KS] without a proof. It is completely covered by our Theorem $3.2((\mathrm{~A}) \Leftrightarrow(\mathrm{B}))$ and our next theorem.

THEOREM 3.3. Under assumptions and notations of Theorem 3.2, the following two conditions are equivalent:

(1) There exists $\varepsilon>0, p>0$ and a subsequence $\left(n_{k}\right)$ such that for some $i$, $1 \leq i \leq d$,

$$
\liminf _{k \rightarrow \infty} \operatorname{Pr}\left\{p \leq \sum_{j=1}^{d}\left(M_{n_{k}}\right)_{i j} \leq \frac{1}{p}\right\} \geq \varepsilon .
$$

(2) $P^{n} \rightarrow Q$ weakly and $Q(I)=1$. 
It is relevant and useful to give first a semigroup version of Theorem 3.3, which will make this theorem crystal clear when $S \subset J^{c}$.

THEOREM 3.4. Let $P$ be a probability measure on a locally compact second countable Hausdorff topological semigroup $S$ such that $S=\operatorname{cl}\left(\bigcup_{n=1}^{\infty} S_{P}^{n}\right)$. Suppose the following condition holds:

For compact subsets $A$ and $B$, the sets

$$
\begin{aligned}
& A B^{-1}=\{y: y x \in A \text { for some } x \in B\}, \\
& A^{-1} B=\{y: x y \in B \text { for some } x \in A\}
\end{aligned}
$$

are compact. Suppose also that there is a compact subset $K_{0} \subset S$, a subsequence $\left(n_{k}\right)$ and $\varepsilon>0$ such that

$$
P^{n_{k}}\left(K_{0}\right)>\varepsilon \quad \text { for each } k \geq 1 .
$$

Then the sequence $\left(P^{n}\right)$ is tight.

ProOF. Let $H=\left\{x \in S: \sum_{n=1}^{\infty} P^{n}\left(N_{x}\right)=\infty\right.$ for every open set $N_{x}$ containing $x$. By (3.5), $H$ is nonempty. Also, $H$ is an ideal of $S$, that is, $H S \subset H$ and $S H \subset H$. By Theorem 5.9 [MT, p. 191], $H$ is a closed completely simple kernel of $S$. [Note that the conditions $(C R)$ and $(C L)$ mentioned in the theorem there are implied by (3.4).] Now we show that

$$
\sup _{K \text { compact }} \lim _{n \rightarrow \infty} \sup _{x \in S} P^{n}\left(K x^{-1}\right)=1 .
$$

The idea of the proof is not new. See Theorem 4.14, p. 51 in [MT]. For completeness, we prove (3.6). Let $b$ be the sup in (3.6). Then $b>0$ by (3.5). Suppose, if possible, that $b<1$. Choose $c=b+[b(1-b) /(1+2 b)]$. Then $b<c<1$ and $c(1+c)<2 b$. Let $B$ be any compact set. There exist a positive integer $N$, a compact set $A$ and $0<q<c / 2$ such that

$$
P^{N}(S-A)<c / 2-q
$$

and

$$
\sup _{z \in S} P^{N}\left(B z^{-1}\right)<c-q
$$

Let $E=A^{-1} B$. Then $E$ is compact by (3.4). For $n>N$ and any $x \in S$,

$$
\begin{aligned}
P^{n}\left(B x^{-1}\right) & =\int P^{N}\left(B x^{-1} y^{-1}\right) P^{n-N}(d y)=\int_{E x^{-1}}+\int_{S-E x^{-1}} \\
& \leq(c-q) P^{n-N}\left(E x^{-1}\right)+(c / 2-q)\left[1-P^{n-N}\left(E x^{-1}\right)\right] \\
& \leq\left(c+c^{2}\right) / 2<b .
\end{aligned}
$$

This is a contradiction. This proves (3.6).

By (3.6), there exist a compact set $K$, elements $a_{n}$ and a positive integer $n_{0}$ such that for $n>n_{0}$,

$$
P^{n}\left(K a_{n}^{-1}\right)>1-\varepsilon / 2 .
$$

By (3.4) and (3.9), $n_{k}>n_{0} \Rightarrow K a_{n_{k}}^{-1} \cap K_{0} \neq \varnothing$. This means that $a_{n_{k}} \in K_{0}^{-1} K$. Thus, if $B=K\left(K_{0}^{-1} K\right)^{-1}$, then $B$ is compact by (3.4) and for $n_{k}>n_{0}$,

$$
P^{n_{k}}(B)>1-\varepsilon / 2 \text {. }
$$


Thus, $\left(P^{n_{k}}\right)$ is tight. Now by employing exactly the same method as given in Step III of the proof of Theorem 4.14 ([MT, p. 50]), it follows that there is a subsequence $\left(m_{k}\right) \subset\left(n_{k}\right)$ such that

$$
P^{m_{k+1}-m_{k}} \rightarrow Q=Q^{2} \in P(S) \quad \text { weakly. }
$$

For $x \in S_{Q}$ and any open $N_{x}$ containing $x$,

$$
0<Q\left(N_{x}\right) \leq \liminf _{k \rightarrow \infty} P^{m_{k+1}-m_{k}}\left(N_{x}\right)
$$

Thus, $S_{Q} \subset H$. Note also that $P * Q=Q * P$ so that $\overline{S_{P} S_{Q}}=\overline{S_{Q} S_{P}}$. This means that $\overline{S S_{Q}}=\overline{S_{Q} S} \subset H$, since $H$ is an ideal and $S_{Q} \subset H$. Since $\operatorname{cl}\left(S S_{Q}\right)$ is also an ideal of $S$ and $H$ is simple, $H=\operatorname{cl}\left(S S_{Q}\right)$. Thus, the closed completely simple semigroup $H$ is generated by the support of $P * Q$. Moreover,

$$
(P * Q)^{m_{k+1}-m_{k}}=P^{m_{k+1}-m_{k}} * Q \rightarrow Q * Q=Q
$$

so that $(P * Q)^{n}$ does not converge to zero vaguely. By Theorem 4.14 ([MT, p. 50]), the group factor of $H$ is compact. Also, it follows easily that given $\varepsilon>0$, there is a compact set $D \subset H$ and a positive integer $m_{0}$ such that for $n \geq m_{0}$, $P^{n} * Q(D)=(P * Q)^{n}(D)>1-\varepsilon$. Thus, if $C$ is compact so that $Q(C)>1-\varepsilon$, then for $n \geq m_{0}, P^{n}\left(D C^{-1}\right)>1-2 \varepsilon$. Thus, $\left(P^{n}\right)$ is tight.

Let us remark that when $S$ is a closed semigroup of nonnegative matrices, then (3.4) holds for $A \subset J^{c}$ and $B \subset J^{c}$. Thus, when $S \subset J^{c}$, Theorem 3.3 is immediate from Theorem 3.4. When $S \cap J \neq \varnothing$, there will be complications in the proof of Theorem 3.3, but the main ideas are those we came across in Theorem 3.4. In what follows, we prove Theorem 3.3 completely. Let $P$ and $S$ be as in Theorem 3.3. Instead of assuming (3.3), we assume the weaker condition

$$
P^{n_{k}}\left(B\left(q_{1}, q_{2}\right)\right) \geq \varepsilon, \quad \text { for each } k \geq 1,
$$

where $q_{1}>0, q_{2}>0$ and $\varepsilon>0$, and

$$
B\left(q_{1}, q_{2}\right)=\left\{A: \max _{i, j} A_{i j} \geq q_{2} \text { and for each } s, \min _{i} A_{i s} \leq q_{1}\right\} .
$$

Write $I^{u}=\left\{A: 1 / u \leq A_{i j} \leq u\right.$ for every $\left.i, j\right\}$. Then using (3.1), we see that there exist a positive integer $N$ and $v>0$ such that

$$
P^{N}\left(I^{v}\right)>1-\varepsilon / 4 \text {. }
$$

Using (3.13) and (3.14), we have for $n_{k}>N$,

$$
\begin{aligned}
\varepsilon & \leq P^{n_{k}}\left(B\left(q_{1}, q_{2}\right)\right)=\int P^{n_{k}-N}\left(x^{-1} B\left(q_{1}, q_{2}\right)\right) P^{N}(d x) \\
& \leq P^{n_{k}-N}\left(\left(I^{v}\right)^{-1} B\left(q_{1}, q_{2}\right)\right)+\varepsilon / 4 .
\end{aligned}
$$

Now it can be easily verified that

$$
\begin{gathered}
\left(I^{v}\right)^{-1} B\left(q_{1}, q_{2}\right) \subset F\left(q_{1}, q_{2}, v\right), \\
F\left(q_{1}, q_{2}, v\right)=\left\{A \in S: q_{2} / v d \leq \max _{i, j} A_{i j} \leq q_{1} v\right\} .
\end{gathered}
$$

Thus, it follows that for $n_{k}>N$,

$$
P^{n_{k}-N}\left(F\left(q_{1}, q_{2}, v\right)\right) \geq 3 \varepsilon / 4 .
$$


Now one can also verify that there are $a>0$ and $b>0$ such that

$$
I^{v} \cdot F\left(q_{1}, q_{2}, v\right) \cdot I^{v} \subset\left\{A \in S: a \leq A_{i j} \leq b \text { for all } i, j\right\} .
$$

Let us call the set on the right $W$. Then it follows from (3.14) and (3.15) that for $n_{k}>N$,

$$
P^{n_{k}+N}(W) \geq(1-\varepsilon / 4)^{2} \cdot(3 \varepsilon / 4) .
$$

Now we need the following lemma.

LEMMA 3.1. Suppose that $W \subset I, W$ is compact and $P^{n_{k}}(W)>q>0$, for each $k \geq 1$. Then $\left(P^{n_{k}}\right)$ is a tight sequence. Also, given $\varepsilon>0$, there is a positive integer $n_{0}$ and a compact set $W_{\varepsilon} \subset I$ such that $\lim _{k} \sup P^{n_{k}+n_{0}}\left(W_{\varepsilon}\right)>1-\varepsilon$.

ProOF. Let $1 \leq i \leq d$. Write

$$
\beta_{u}=\left\{A \in S: u \leq \sum_{j=1}^{d} A_{i j} \leq \frac{1}{u}\right\} .
$$

We first show that

$$
\sup _{u>0} \lim _{n \rightarrow \infty} \sup _{x \in S} P^{n}\left(x^{-1} \beta_{u}\right)=1 .
$$

Notice that the set $W$ is contained in some $\beta_{u}$. It can also be verified easily that for each $u>0$, the set $\beta_{u}\left(I^{v}\right)^{-1}$ is contained in $\beta_{u^{\prime}}$, for some $u^{\prime}>0$. The proof of (3.17) can now follow exactly the same lines as in the proof of (3.6). Here we only use

$$
\begin{aligned}
P^{n}\left(x^{-1} \beta_{u}\right) & =\int P^{N}\left(y^{-1} x^{-1} \beta_{u}\right) P^{n-N}(d y) \\
& =\int_{x^{-1} \beta_{u^{\prime}}}+\int_{S-x^{-1} \beta_{u^{\prime}}} .
\end{aligned}
$$

The details are the same as before. So we may and do take (3.17) for granted. By (3.17), given $0<\varepsilon<q$, there exist $u>0$ and elements $x_{m}$ in $S$ such that for all sufficiently large $m$ and $k$,

$$
P^{m}\left(x_{m}^{-1} \beta_{u}\right)>1-\varepsilon / 2 \quad \text { and } \quad P^{n_{k}}(W)>q .
$$

It follows that for sufficiently large $k, x_{n_{k}}^{-1} \beta_{u} \cap W \neq \varnothing$ so that $x_{n_{k}} \in \beta_{u} W^{-1}=T_{u}$, say.

It is now routine to verify the following fact. There exist $a_{\varepsilon}>0$ and $b_{\varepsilon}>0$ such that

$$
T_{u}^{-1} \beta_{u} \subset L_{\varepsilon} \subset B_{\varepsilon}
$$

where

$$
L_{\varepsilon}=\left\{A \in S: \max _{i, j} A_{i j} \geq a_{\varepsilon} \text { and } \min _{i, j} A_{i j} \leq b_{\varepsilon}\right\}
$$

and

$$
B_{\varepsilon}=\left\{A \in S: \min _{i, j} A_{i j} \leq b_{\varepsilon}\right\} .
$$

Thus for each $\varepsilon>0$, there exists $k_{0}$ such that for $k \geq k_{0}$,

$$
P^{n_{k}}\left(B_{\varepsilon}\right) \geq P^{n_{k}}\left(L_{\varepsilon}\right)>1-\varepsilon .
$$


Using (3.14) and the same proof used for $(B) \Rightarrow(C)$ in Theorem 3.2 , we see that given $0<u^{\prime}<1$, taking $u=u^{\prime}(1-\varepsilon / 4)^{2}$,

$$
\begin{aligned}
(1- & \varepsilon / 4)^{2}\left(1-u^{\prime}\right) \\
& \leq \operatorname{Pr}\left(M_{2 N+\left(n_{k}-2 N\right)} \in B_{u}, M_{N} \in I^{v} \text { and } A_{N+n_{k}+1} \cdots A_{2 N+n_{k}} \in I^{v}\right) \\
& \leq(1-\varepsilon / 4)^{2} \cdot P^{n_{k}-2 N}\left(M_{u}\right),
\end{aligned}
$$

where

$$
M_{u}=\left\{D \in S: \max _{i, j} D_{i j} \leq b_{u} v^{2}\right\} .
$$

It follows that $\left(P^{n_{k}}\right)$ is a tight sequence.

Now we note that closure $\left(I^{v} \cdot L_{\varepsilon} \cdot I^{v}\right) \subset I$ so that it follows from (3.14), (3.19) and the tightness of $\left(P^{n_{k}}\right)$ that there is a compact set $W_{\varepsilon} \subset I$ such that for sufficiently large $k$,

$$
P^{n_{k}+2 N}\left(W_{\varepsilon}\right)>(1-\varepsilon)^{3} .
$$

Now we continue with the hypothesis of Lemma 3.1. By (3.19), there is a subsequence $m_{k} \subset\left(n_{k}\right)$ such that $P^{m_{k}} \rightarrow P^{\prime}$ weakly, where $P^{\prime}$ is a probability measure different from $\delta_{0}$. Following the same proof as given in Step III (of Theorem 7.14, p. 54 in $[\mathbf{M T}])$, we see that there is a subsequence $\left(p_{k}\right) \subset\left(m_{k}\right)$ such that for $j \geq 1$,

$$
P^{p_{k}-j} \rightarrow P_{j} \in P(S), \quad P_{0}=P^{\prime}
$$

(Note that $P_{j} \in P(S)$, since $\left(P^{p_{k}-j}\right)$ is tight. Because of the tightness of $\left(P^{p_{k}}\right)$ and the regularity of $P^{j}$, given $\varepsilon>0$, there are compact sets $D_{\varepsilon}$ and $E_{\varepsilon}$ with $E_{\varepsilon} \subset J^{c}$ such that

$$
\begin{aligned}
1-\varepsilon & <P^{p_{k}}\left(D_{\varepsilon}\right)=\int P^{p_{k}-j}\left(D_{\varepsilon} x^{-1}\right) P^{j}(d x) \\
& =\int_{E_{\varepsilon}}+\int_{S-E_{\varepsilon}} \leq P^{p_{k}-j}\left(D_{\varepsilon} E_{\varepsilon}^{-1}\right)+\varepsilon .
\end{aligned}
$$

Since $E_{\varepsilon} \subset J^{c}, D_{\varepsilon} E_{\varepsilon}^{-1}$ is compact.) Thus, for each $j \geq 1, P_{j} * P^{j}=P^{j} * P_{j}=P^{\prime}$. Now we show that $\left(P_{p_{k}}\right)$ is tight. Notice that

$$
P_{p_{k}} * P^{p_{k}+2 N}=P^{p_{k}+2 N} * P_{p_{k}}=P^{\prime} * P^{2 N} \text {. }
$$

We use (3.20). Given $\varepsilon>0$, there is a compact set $W_{\varepsilon} \subset I$ such that $P^{\prime} * P^{2 N}\left(W_{\varepsilon}\right)>$ $1-3 \varepsilon$ and (3.20) holds so that

$$
\begin{aligned}
& 1-3 \varepsilon<P^{\prime} * P^{2 N}\left(W_{\varepsilon}\right)=\int P_{p_{k}}\left(x^{-1} W_{\varepsilon}\right) P^{p_{k}+2 N}(d x) \\
&=\int_{W_{\varepsilon}}+\int_{S-W_{\varepsilon}} \leq P_{p_{k}}\left(W_{\varepsilon}^{-1} W_{\varepsilon}\right)+3 \varepsilon .
\end{aligned}
$$

Since $W_{\varepsilon} \subset I, W_{\varepsilon}^{-1} W_{\varepsilon}$ is compact and $\left(P_{p_{k}}\right)$ is tight. If $Q$ is a weak*-cluster point of $\left(P_{p_{k}}\right)$, then $Q \in P(S)$ and it follows as in the proof of Step III of Theorem 7.14 [MT] that $Q=Q^{2}$ and there is a subsequence $\left(q_{k}\right) \subset\left(p_{k}\right)$ such that

$$
P^{q_{k+1}-q_{k}} \rightarrow Q=Q^{2} \in P(S) .
$$

Notice that by (3.22) and by a similar argument,

$$
Q\left(W_{\varepsilon}^{-1} W_{\varepsilon} \cap W_{\varepsilon} W_{\varepsilon}^{-1}\right)>1-12 \varepsilon .
$$


Since $W_{\varepsilon} \subset I, W_{\varepsilon}^{-1} W_{\varepsilon} \cap W_{\varepsilon} W_{\varepsilon}^{-1} \subset J^{c}$. It follows that

$$
Q\left(J^{c}\right)=1 .
$$

Write $s_{k}=q_{k+1}-q_{k}$ and $E_{v}=\left\{A \in S: \max _{i, j} A_{i j} \geq v\right\}$. By (3.23) and (3.24), there is a positive $v$ such that for sufficiently large $k, P^{s_{k}}\left(E_{v}\right)>1-\varepsilon$. As in (3.14), there are positive integers $N, r, v^{\prime}$ such that $P^{N}\left(I^{r}\right)>1-\varepsilon$ and $P^{2 N}\left(I^{v^{\prime}}\right)>1-\varepsilon$. Then

$$
1-\varepsilon<P^{s_{k}}\left(E_{v}\right) \leq P^{s_{k}-2 N}\left(\left(I^{v^{\prime}}\right)^{-1} E_{v}\right)+\varepsilon .
$$

Since $\left(I^{v^{\prime}}\right)^{-1} E_{v} \subset E_{w}$ for some $w>0$, for sufficiently large $k$, then

$$
P^{s_{k}-2 N}\left(E_{w}\right)>1-2 \varepsilon \text {. }
$$

Since $D \equiv \operatorname{closure}\left(I^{r} \cdot E_{w} \cdot I^{r}\right) \subset I$, for large $k$

$$
P^{s_{k}}(D)>(1-\varepsilon)^{2}(1-2 \varepsilon) \text {. }
$$

This means that

$$
Q(I)=1 .
$$

Now let $x, y \in S \cap J^{c}$ be such that $x \notin S y$. Then it is not difficult to show that there exist open subsets $N_{x} \subset J^{c}, N_{y} \subset J^{c}$ such that $x \in N_{x}, y \in N_{y}$ and $S \cap\left(N_{x} N_{y}^{-1}\right)=\varnothing$. By (3.16), there exists $x \in I \cap S$ such that for every open set $N_{x}$ containing $x$,

$$
P^{n}\left(N_{x}\right) \nrightarrow 0 \text { as } n \rightarrow \infty .
$$

Following the proof of Theorem 5.9 [MT] almost word for word, we have

$$
y \in S x \cap J^{c} \Rightarrow S x=S y
$$

and

$$
y \in x S \cap J^{c} \Rightarrow x S=y S .
$$

Notice that if we write $S_{1}=S-\{0\}$, then since $x \in I$, we have

$$
\begin{aligned}
x S x & =x S_{1} x \cup\{0\} & & \text { if } 0 \in S ; \\
& =x S_{1} x & & \text { if } 0 \notin S .
\end{aligned}
$$

In any case, $x S_{1} x \subset I$ and $x S_{1} x$ is a subsemigroup. Let $y \in x S_{1} x$. Using (3.28),

$$
y \cdot x S x=x S x \text { and therefore } y x S_{1} x=x S_{1} x .
$$

Similarly, using (3.27),

$$
x S_{1} x y=x S_{1} x \quad \text { for } y \in x S_{1} x .
$$

By (3.29) and (3.30), $x S_{1} x$ is a group contained in $I$.

Let us now define the set $K_{0}$ by

$$
K_{0}=\left\{y \in I \cap S: P^{n}\left(N_{y}\right) \nrightarrow 0 \text { as } n \rightarrow \infty \text { for every open } N_{y} \text { containing } y\right\} .
$$

Then $K_{0}$ is a nonempty ideal of $S \cap J^{c}$. Also, by what we have proven above, for each $y \in K_{0}, y^{3} \in y S_{1} y$, which is a group. Thus, by $[\mathbf{C}], m\left(K_{0}\right)$ is a completely simple minimal ideal of $K_{0}$. Since every idempotent in $I$ has rank one and since by 
(3.28), $y^{3} \in y S \Rightarrow y \in y^{3} S \subset y S$ and since every element in a group has the same rank, it follows that $m\left(K_{0}\right)=K_{0}$.

Since $Q(I)=1$ and for every $y \in S_{Q}$ and any open $N_{y}$ containing $y$,

$$
0<Q\left(N_{y}\right) \leq \liminf _{k} P^{s_{k}}\left(N_{y}\right),
$$

it is clear that

$$
Q\left(K_{0}\right)=1
$$

Since $P * Q=Q * P$ and $Q=Q^{2}$,

$$
\begin{aligned}
(P * Q)^{n}\left(K_{0}\right) & =\left(P^{n} * Q\right)\left(K_{0}\right) \geq\left(P^{n} * Q\right)\left(\left(S \cap J^{c}\right) K_{0}\right) \\
& \geq P^{n}\left(S \cap J^{c}\right) Q\left(K_{0}\right)=1 \quad \text { for every } n \geq 1 .
\end{aligned}
$$

Restricting $Q$ and $P * Q$ to $K_{0}$ (with relative topology) and noticing that $\operatorname{cl}\left(S S_{Q}\right)$ is an ideal of $S$ (since $P * Q=Q * P$ ) so that $K_{0} \subset \operatorname{cl}\left(S S_{Q}\right)$. This means that the support of $P * Q$ restricted to $K_{0}$ generates $K_{0}$. It follows from Theorem 4.14 in [MT] that the group factor of $K_{0}$ is compact in $K_{0}$. Since $K_{0} \subset I$, the group factor of $K_{0}$ is a singleton. This means that $(P * Q)^{n}=P^{n} * Q$ converges weakly to some $P_{0}$, a probability measure. Thus, given $\varepsilon>0$, there is a compact set $L$ and a compact $E$, both contained in $I$ such that for sufficiently large $n$,

$$
1-\varepsilon<P^{n} * Q(L)<\int_{E} P^{n}\left(L z^{-1}\right) Q(d z)+\varepsilon .
$$

Thus, for large $n, P^{n}\left(L E^{-1}\right)>1-2 \varepsilon$. Thus, $\left(P^{n}\right)$ is tight and we have proven Theorem 3.3.

For nonnegative matrices, we can also prove the following theorem (along the lines of $[\mathbf{K S}]$, but more general than what is given in $[\mathbf{K S}]$ ).

THEOREM 3.5. Let $P$ and $S$ be as in Theorem 3.2. Suppose that $U \in P(S)$, $P * U=U$ and $U(\{B \in S: B$ has a zero column $\})=0$. Then $P^{n} \rightarrow Q$ weakly, $Q(I \cap S)=1, U * U=U, Q * U=U, U * Q=Q$ and $S_{U} \subset S_{Q}$.

4. Weak convergence in $2 \times 2$ real matrices. Let $P$ be a regular probability measure on $2 \times 2$ real matrices and $S$ be the closed semigroup with respect to multiplication generated by $S_{P}$. Suppose $P^{n} \rightarrow Q$ weakly. Then $S_{Q}$ is the completely simple kernel of $S$ and $Q=Q * P=P * Q$. Also, $S_{Q}=m(S)$ and the group factor of $S_{Q}$ is compact. There are now two possibilities:

(I) The rank of the matrices in $m(S)$ is two. Then $S_{Q}$ has only one idempotent element and it is a compact group. Since $S \cdot S_{Q} \subset S_{Q}, S=S_{Q}$. Thus, in this case, the closed semigroup generated by $S_{P}$ is a compact group and $Q$ is the normed Haar measure on this group.

(II) $S_{Q} \subset$ rank one matrices. In this case we also assume that $m(S)$ has no subgroup with exactly two elements, so any compact subgroup of $m(S)$ is a singleton. [Note that such a condition was not needed in $\S 3$, since a compact group of positive matrices is a singleton.] Under these assumptions, for any idempotent $e$ in $S_{Q}$, $e S_{Q} e$ is a compact subgroup of $S_{Q}$ and therefore, a singleton. Since $S_{Q}=S_{Q} e S_{Q}$, every $x$ in $S_{Q}$ is of the form yez so that

$$
x^{2}=y(e z y e) z=y e z=x .
$$

Thus, every element in $S_{Q}$ has rank one and is idempotent. 
Now let us make the following assumption:

$$
S_{Q} \cap J^{c} \neq \varnothing .
$$

(Recall that $J^{c}$ is the set of all $2 \times 2$ matrices which do not have any zero rows or columns.) Let us choose $e$ from $S_{Q} \cap J^{c}$ so that $e$ can be taken as

$$
e=\left(\begin{array}{ll}
a_{1} e_{1} & a_{1} e_{2} \\
a_{2} e_{1} & a_{2} e_{2}
\end{array}\right)
$$

where

$$
a_{1}, a_{2}, e_{1}, e_{2} \text { are all nonzero and } a_{1} e_{1}+a_{2} e_{2}=1 .
$$

(The last equality holds because $e=e^{2}$.) Recall that

$$
e S e=e S_{Q} e=e .
$$

We now show that

$$
\text { either } e S=e S_{Q}=e \text { or } \quad S e=S_{Q} e=e \text {. }
$$

(In other words, for any $e$ in $S_{Q} \cap J^{c}$, either every row of $e$ is a common left eigenvector for every matrix in $S$ with eigenvalue 1 or every column of $e$ is a common right eigenvector for every matrix in $S$ with eigenvalue 1.) Notice that $e=e^{2} \in S_{Q}$ which is an ideal of $S$ so that $e S_{Q} \subset e S=e(e S) \subset e S_{Q}$. Similarly, $S_{Q} e=S e$. To establish (4.5), let $y \in e S_{Q}$ and $x \in S_{Q} e$ such that

$$
x=\left(\begin{array}{ll}
b_{1} x_{1} & b_{1} x_{2} \\
b_{2} x_{1} & b_{2} x_{2}
\end{array}\right) \quad \text { and } y=\left(\begin{array}{ll}
c_{1} y_{1} & c_{1} y_{2} \\
c_{2} y_{1} & c_{2} y_{2}
\end{array}\right),
$$

where

$$
b_{1} x_{1}+b_{2} x_{2}=1 \quad \text { and } \quad c_{1} y_{1}+c_{2} y_{2}=1 .
$$

Since $y x=e$ by (4.4) and $e \in J^{c}, y$ cannot have a zero row and $x$ cannot have a zero column. Therefore,

$$
c_{1} \neq 0, \quad c_{2} \neq 0, \quad x_{1} \neq 0, \quad x_{2} \neq 0 .
$$

Now we distinguish between three possible cases.

Case 1. Suppose that there is an $x \in S_{Q} e$ for which $b_{1}=0$. Since $y x=e$, simple matrix multiplication yields that $c_{1} y_{2}=a_{1} e_{2}$ and $c_{2} y_{2}=a_{2} e_{2}$. Using (4.3) and (4.7), it follows easily that $y=e$. Thus, in this case, $e S=e$.

Case 2. Suppose that for each $x$ in $S_{Q} e, b_{1}$ is nonzero, but there exists $y \in S_{Q}$ such that $y_{2}=0$. Then for this $y$ in $e S_{Q}$ and any $x$ in $S_{Q} e, y x=e$ implies (after a little computation) that $x=e$. Thus, in this case, $S e=e$.

Case 3. Suppose that for each $x \in S_{Q} e, b_{1}$ is nonzero and for each $y$ in $e S_{Q}$, $y_{2} \neq 0$. Then, for our convenience, we rewrite $y, x$ and $e$ ( $y$ arbitrarily chosen from $e S_{Q}$ and $x$ from $x S_{Q}$ ) as

$$
y=\left(\begin{array}{cc}
a & b \\
\frac{a(1-a)}{b} & 1-a
\end{array}\right), \quad x=\left(\begin{array}{cc}
c & d \\
\frac{c(1-c)}{d} & 1-c
\end{array}\right), \quad e=\left(\begin{array}{cc}
k^{\prime} & k \\
\frac{k^{\prime}\left(1-k^{\prime}\right)}{k} & 1-k^{\prime}
\end{array}\right),
$$

where $b \neq 0, d \neq 0, k \neq 0$. Now simple computations using $y x=e$ give

$$
\begin{gathered}
a d+b(1-c)=k, \quad(c / d)[a d+b(1-c)]=k ; \\
\{(1-a) / b\}[a d+b(1-c)]=1-k^{\prime} .
\end{gathered}
$$


Simplifying the equation in (4.9), we have

$$
(k-d)(k-b)=0 .
$$

If $k=b$, then we have from (4.9) that

$$
c / d=a / b=k^{\prime} / k
$$

which means that $y=e$. This means that if there is an $x$ in $S_{Q} e$ such that $x \neq e$, then $e S_{Q}=e S=e$. Similarly, if $k=d,(4.5)$ must again hold.

Thus, we have established (4.5) in all possible cases under the assumption of (4.1). Now we consider the case when (4.1) no longer holds. We still assume, of course, the condition of (II). We will show that even in this case, (4.5) holds.

Recall that by (II), every element in $S_{Q}$ is still idempotent. First, let

$$
e \in S_{Q} \quad \text { and } \quad e=\left(\begin{array}{ll}
0 & 0 \\
0 & 1
\end{array}\right)
$$

Then

$$
S e=S_{Q} e \subset\left\{\left(\begin{array}{ll}
0 & r \\
0 & 1
\end{array}\right): r \text { a real number }\right\}
$$

and

$$
e S=e S_{Q} \subset\left\{\left(\begin{array}{ll}
0 & 0 \\
r & 1
\end{array}\right): r \text { a real number }\right\} .
$$

Using the fact that $e S_{Q} e=e$, it follows after computations that either

$$
e S=e S_{Q}=e \quad \text { and } \quad S \subset\left\{\left(\begin{array}{cc}
s & t \\
0 & 1
\end{array}\right): s, t \text { reals }\right\}
$$

or

$$
S e=S_{Q} e=e \quad \text { and } S \subset\left\{\left(\begin{array}{cc}
s & 0 \\
t & 1
\end{array}\right): s, t \text { reals }\right\} .
$$

Next, let $e \in S_{Q}$ be of the form $\left(\begin{array}{ll}0 & r \\ 0 & 1\end{array}\right), r \neq 0$.

It can be shown again that in this case either

$$
S e=S_{Q} e=e \quad \text { or } \quad e S=e S_{Q}=e
$$

and

$$
S \subset\left\{\left(\begin{array}{cc}
s & t \\
0 & 1
\end{array}\right): s, t \text { reals }\right\} .
$$

There is no point into going into every other possible case. Let us just mention that it can be verified in exactly the same manner that (4.5) holds in all the other cases. Thus, we have proven the following Kesten-Spitzer type theorem for real $2 \times 2$ matrices.

THEOREM 4.1. Let $P$ be a probability measure on $2 \times 2$ real matrices and $S$ be the closed semigroup generated by $S_{P}$. Then the following results hold:

(a) If $P^{n} \rightarrow Q \in P(S)$ weakly, then

(i) $S_{Q}=m(S)$;

(ii) $Q=\delta_{0}$ iff $0 \in S$;

(iii) the minimal rank of the matrices in $S$ is two iff $S$ is a compact group of invertible matrices and $Q$ is the normed Haar measure on $S$; 
(iv) the minimal rank of the matrices in $S$ is one and $m(S)$ contains no subgroup with exactly two elements iff there is a nonzero idempotent rank one matrix $x$ in $S$ such that $x S=x$ or $S x=x$.

(b) Suppose that $\lim _{n \rightarrow \infty} P^{n}(G)=1$ for every open set $G \supset m(S)$. If the rank of the matrices in $m(S)$ is two, then

(i) $P^{n} \rightarrow 0$ vaguely when $S$ is noncompact;

(ii) when $S$ is compact, $S$ is a compact group and then $P^{n}$ converges weakly to the normed Haar measure on $S$ iff there is no proper normal subgroup $H$ of $S$ such that $S_{P} \subset g H$ for some $g \in S-H$. If the rank of the matrices in $m(S)$ is one, then $P^{n} \rightarrow Q$ weakly and $S_{Q}=m(S)$ when there is a nonzero vector $x$ such that $x S_{P}=x$ or $S_{P} x^{T}=x^{T}$.

5. The convergence problem in real matrices. Here we derive analogs of earlier results in $d \times d$ matrices with arbitrary real entries for arbitrary finite $d$. First, we need a lemma.

LEMMA 5.1. Suppose that $x=x^{2}$ is a $d \times d$ real matrix with no zero row. Suppose that there are two rank one $d \times d$ real matrices $y$ and $z$ such that

$$
x y=x \quad \text { and } \quad x z=x .
$$

Then, $y=y^{2}, z=z^{2}$ and $y z=y$.

PROOF. Notice that $x$ has rank one. Write $x$ as $x=\left(x_{i j}\right)=\left(a_{i} x_{j}\right)$, where each $a_{i}$ is nonzero (since $x$ has no zero row) and not all the $x_{j}$ 's are zero. Write $y$ as $y=\left(y_{i j}\right)=\left(b_{i} y_{j}\right)$. Since $x y=x$, we have for $1 \leq i, j \leq d$,

$$
a_{i} x_{j}=\sum_{k=1}^{d} a_{i} x_{k} b_{k} y_{j}
$$

Since $x=x^{2}$ and $x \neq 0$,

$$
\sum_{i=1}^{d} a_{i} x_{i}=1
$$

It follows from (5.2) that

$$
\sum_{i=1}^{d} a_{i} x_{i}=\left(\sum_{i=1}^{d} a_{i} y_{i}\right) \cdot\left(\sum_{k=1}^{d} b_{k} x_{k}\right) .
$$

Cancelling $a_{i}$ and then multiplying by $b_{j}$ in (5.2), we have

$$
b_{j} x_{j}=b_{j} y_{j} \cdot \sum_{k=1}^{d} b_{k} x_{k}
$$

so that

$$
\sum_{j=1}^{d} b_{j} x_{j}=\left(\sum_{j=1}^{d} b_{j} y_{j}\right)\left(\sum_{k=1}^{d} b_{k} x_{k}\right)
$$


It follows from (5.3), (5.4) and (5.5) that

$$
\sum_{k=1}^{d} b_{k} x_{k} \neq 0 \text { and } \sum_{j=1}^{d} b_{j} y_{j}=1 .
$$

This means that $y=y^{2}$.

Similarly, we write $z$ as $z=\left(z_{i j}\right)=\left(c_{i} z_{j}\right)$. Since $x z=x$, it follows as before that $z=z^{2}$. It follows from (5.2) that

$$
y_{j}=0 \Rightarrow x_{j}=0
$$

also, $x_{j} / y_{j}=\sum_{k=1}^{d} b_{k} x_{k}$.

Let us now prove that $y z=y$. First, notice that

$$
(y z)_{i j}=b_{i} z_{j} \cdot \sum_{k=1}^{d} c_{k} y_{k} .
$$

As in (5.7), we also have

$$
x_{j}=0, \quad \text { whenever } z_{j}=0
$$

and

$$
x_{j} / z_{j}=\sum_{k=1}^{d} c_{k} x_{k}, \quad \text { whenever } z_{j} \neq 0 .
$$

Suppose that $y_{j}=0$. Then $x_{j}=0$ by (5.7). Since $x z=x$,

$$
a_{i} x_{j}=a_{i} z_{j} \cdot \sum_{k=1}^{d} b_{k} x_{k}
$$

and therefore, since by $(5.6), \sum_{k=1}^{d} b_{k} x_{k} \neq 0$, we have $z_{j}=0$. Then $(y z)_{i j}=0$ by (5.8). Thus, when $y_{j}=0,(y z)_{i j}=y_{i j}\left(=b_{i} y_{j}\right)$.

Now suppose that $y_{j} \neq 0$. By (5.2) and (5.6),

$$
x_{j}=y_{j} \cdot \sum_{k=1}^{d} b_{k} x_{k} \neq 0 .
$$

Therefore, by (5.9), $z_{j} \neq 0$ and

$$
0 \neq x_{j}=z_{j} \cdot \sum_{k=1}^{d} c_{k} x_{k} .
$$

It follows also from (5.2) that

$$
\sum_{s=1}^{d} c_{s} x_{s}=\left(\sum_{s=1}^{d} c_{s} y_{s}\right) \cdot\left(\sum_{k=1}^{d} b_{k} x_{k}\right)
$$

By (5.10)-(5.12),

$$
y_{j}=z_{j} \cdot \sum_{s=1}^{d} c_{s} y_{s}
$$


By (5.8) and (5.13),

$$
(y z)_{i j}=b_{i} y_{j}=y_{i j}
$$

This proves that $y z=y$.

In [KS], it was shown for $2 \times 2$ nonnegative matrices that under $\left(K S_{1}\right)$ and $\left(K S_{2}\right)$ (see Theorem 4.1), whenever $P^{n} \rightarrow Q$ weakly and $Q \neq \delta_{0}$, then there exists $x=\left(x_{1}, x_{2}\right), x_{1}>0, x_{2}>0$, such that either $x S_{P}=x$ or $S_{P} x^{T}=x^{T}$. Here we show that this result is no longer valid for higher dimensional matrices.

EXAMPLE 5.1. Consider the semigroup $S$ of $3 \times 3$ strictly positive matrices given by $S=\left\{e_{0}, e_{1}, e_{2}, e_{3}\right\}$, where

$$
\begin{array}{ll}
e_{0}=\left(\begin{array}{lll}
\frac{1}{2} & \frac{1}{6} & \frac{1}{3} \\
\frac{1}{2} & \frac{1}{6} & \frac{1}{3} \\
\frac{1}{2} & \frac{1}{6} & \frac{1}{3}
\end{array}\right), & e_{1}=\left(\begin{array}{ccc}
\frac{3}{4} & \frac{1}{12} & \frac{1}{6} \\
\frac{3}{4} & \frac{1}{12} & \frac{1}{6} \\
\frac{3}{4} & \frac{1}{12} & \frac{1}{6}
\end{array}\right), \\
e_{2}=\left(\begin{array}{ccc}
\frac{1}{2} & \frac{1}{6} & \frac{1}{3} \\
1 & \frac{1}{3} & \frac{2}{3} \\
\frac{1}{4} & \frac{1}{12} & \frac{1}{6}
\end{array}\right), & e_{3}=\left(\begin{array}{ccc}
\frac{3}{4} & \frac{1}{12} & \frac{1}{6} \\
\frac{3}{2} & \frac{1}{6} & \frac{1}{3} \\
\frac{3}{8} & \frac{1}{24} & \frac{1}{12}
\end{array}\right) .
\end{array}
$$

Then the multiplication table for $S$ is given by

$\begin{array}{ccccc}\times & e_{0} & e_{1} & e_{2} & e_{3} \\ e_{0} & e_{0} & e_{1} & e_{0} & e_{1} \\ e_{1} & e_{0} & e_{1} & e_{0} & e_{1} \\ e_{2} & e_{2} & e_{3} & e_{2} & e_{3} \\ e_{3} & e_{2} & e_{3} & e_{2} & e_{3}\end{array}$

The semigroup $S$ is completely simple since it is finite and has no proper ideals. Also, every element of $S$ has rank one. Consider the probability measure $P$ on $S$ given by

$$
P\left(e_{i}\right)=1 / 4, \quad i=0,1,2,3 .
$$

(Actually, any $P$ such that $S_{P}=S$ will do.) Since every subgroup of $S$ is a singleton, by Theorem $2.1, P^{n} \rightarrow Q$ and $S_{Q}=S$.

Now suppose that there exists a nonzero vector $u=\left(u_{1}, u_{2}, u_{3}\right)$, at least one $u_{i}$ being nonzero, such that $u S=u$. Choose $a \neq 0, b \neq 0$ and $c \neq 0$ such that $c u_{1}+a u_{2}+b u_{3}=1$. Define the matrix $x$ by

$$
x=\left(\begin{array}{lll}
c u_{1} & c u_{2} & c u_{3} \\
a u_{1} & a u_{2} & a u_{3} \\
b u_{1} & b u_{2} & b u_{3}
\end{array}\right) .
$$

Then $x=x^{2}$ and $x S=x$. It follows then from Lemma 5.1 that for $y, z$ in $S, y z=y$. This contradicts the above multiplication table.

Despite the above example, half of Theorem 3 [KS, p. 384] holds even for general $d \times d(d \geq 2)$ real matrices as we show in Theorem 5.1. It should be pointed out that for a vector $x$ and a set $A$ of matrices, the equation $x A=x$ does no longer imply that $A$ is bounded unless $x$ is strictly positive and the matrices in $A$ nonnegative. Also note that for nonnegative matrices, the conditions $\left(K S_{1}\right)$ and $\left(K S_{2}\right)$ are equivalent to the condition that $\lim _{n \rightarrow \infty} P^{n}(I)=1$. This is because of the way we proved (3.1) and because

$$
P^{2 m}\left(J_{r}\right) \geq \int_{J_{r}} P^{m}\left(x^{-1} J_{r}\right) P^{m}(d x)=P^{m}\left(J_{r}\right)
$$


since $S \subset x^{-1} J_{r}$ for each $x \in J_{r}$, and

$$
P^{2 m}\left(J_{c}\right) \geq \int_{J c} P^{m}\left(J_{c} x^{-1}\right) P^{m}(d x)=P^{m}\left(J_{c}\right)
$$

since $S \subset J_{c} x^{-1}$ for every $x$ in $J_{c}$, where

$$
J_{r}=\{A: A \text { has at least one zero row }\}
$$

and

$$
J_{c}=\{A: A \text { has at least one zero column }\} .
$$

$I$ is also an open ideal of $J^{c}$ containing all rank one matrices in $J^{c}$. Under $\left(K S_{1}\right)$ and $\left(K S_{2}\right)$, for $P^{n}$ to converge weakly to $Q$ in nonnegative matrices, it is necessary that $S_{Q} \subset$ rank one matrices and $\lim _{n \rightarrow \infty} P^{n}(G)=1$ for every open set $G$ containing rank one matrices. Considering all these, it is now clear that condition (5.14) below is for real matrices what conditions $\left(K S_{1}\right)$ and $\left(K S_{2}\right)$ are for nonnegative matrices.

THEOREM 5.1. Let $P$ be a probability measure on $d \times d$ real matrices such that for every open set $G \supset I$, where in real matrices $I$ denotes the set of rank one matrices,

$$
\lim _{n \rightarrow \infty} P^{n}(G)=1
$$

Suppose that for some $x=\left(x_{1}, x_{2}, \ldots, x_{d}\right)$, not all $x_{i}$ 's zero, $x S_{P}=x$ or $S_{p} x^{T}=$ $x^{T}$. Then $P^{n} \rightarrow Q$ weakly and $S_{Q} \subset I$.

ProOF. First we need to prove that $\left(P^{n}\right)$ is tight. Let $S$ be the closed semigroup generated by $S_{P}$. Since $x S=x, S$ has no zero. If $P^{m}(I)=0$ for each positive integer $m$, then noting that $I \cap S$ is a closed subset of $S$ it follows immediately from Lemma 7.7 in $[\mathbf{H M}]$ that $\left(P^{n}\right)$ is tight. Let us then assume that $P^{m}(I)>0$ for some positive integer $m$. Then as in Theorem 4.3, it follows that

$$
\lim _{n \rightarrow \infty} P^{n}(I)=1 \text {. }
$$

Let $x S_{P}=x$, where $x$ is a nonzero vector. Then $x S=x$. There is a rank one $d \times d$ idempotent matrix $x_{0}$ such that $x$ is its first row. Then $x_{0} S=x_{0}$. Since $x_{0} \neq 0$, $0 \notin S$. Now notice that

$$
y, z \in I \cap S \Rightarrow y z=y
$$

by Lemma 5.1. Let $p>0$. There exists $q>0$ and a positive integer $N$ such that

$$
n \geq N \Rightarrow P^{n}(I \cap S)>1-q>\sqrt{1-p} .
$$

Let $K_{p}$ be a compact set $\subset I \cap S$ such that $P^{n}\left(K_{p}\right)>1-q$. Let $n \geq N$. Then using (5.16),

$$
\begin{aligned}
P^{n+N}\left(K_{p}\right) & =\int P^{N}\left(K_{p} y^{-1}\right) P^{n}(d y) \\
& \geq \int_{S \cap I} P^{N}\left(K_{p} y^{-1}\right) P^{n}(d y) \\
& =P^{N}\left(K_{p}\right) P^{n}(S \cap I)>(1-q)^{2}>1-p .
\end{aligned}
$$


This proves that the sequence $\left(P^{n}\right)$ is a tight sequence. By Theorem 4.3 of $[\mathbf{M}]$, $(1 / n) \sum_{k=1}^{n} P^{k} \rightarrow Q \in P(S)$. Since $S \cap I$ is closed, $S_{Q} \subset S \cap I$. Since $x S=x$ and $x$ is nonzero, $S$ cannot contain any subgroup of the form $\{-1,1\}$. It follows that the group factor of $S_{Q}$ being compact is a singleton. By Theorem 4.3 of $[\mathbf{M}]$, $P^{n} \rightarrow Q$ weakly.

EXAMPLE 5.2. Consider the semigroup $S_{0}=K_{1} \cup K_{2}$ of $2 \times 2$ nonnegative matrices, where

$$
K_{1}=\left\{\left(\begin{array}{cc}
1 & m \\
0 & n
\end{array}\right): m, n \text { are nonnegative integers }\right\}
$$

and

$$
K_{2}=\left\{\left(\begin{array}{ll}
1 & n \\
0 & 0
\end{array}\right): n \text { is a nonnegative integer }\right\} .
$$

Notice that the set $K_{2}$ is a right zero semigroup so that for any $e \in K_{2}, e K_{2} e=e$. It is clear that $K_{2}$ is the completely simple kernel of $S_{0}$. Notice that if $x=(1,0)$, then $s_{0} x^{T}=x^{T}$. Consider a probability measure $P$ on $S_{0}$ such that

$$
S_{P} \supset\left\{\left(\begin{array}{ll}
1 & 1 \\
0 & 1
\end{array}\right),\left(\begin{array}{ll}
1 & 1 \\
0 & 0
\end{array}\right)\right\} .
$$

It is clear that Theorem 5.1 applies and $P^{n} \rightarrow Q$ weakly, where $S_{Q} \subset K_{2}$ (actually, $S_{Q}=K_{2}$ if $S_{P}$ contains also $\left.\left(\begin{array}{ll}1 & 0 \\ 0 & 0\end{array}\right)\right)$. Notice that neither $S_{Q}$ nor the closed semigroup generated by $S_{P}$ is compact, unlike in Theorem 3.1.

Finally we state a general theorem for real matrices.

THEOREM 5.2. Let $P$ be a probability measure on $d \times d$ real matrices and $S=\operatorname{cl}\left(\bigcup_{n=1}^{\infty} S_{P}^{n}\right)$. Suppose that for each open set $G \supset m(S), \lim _{n \rightarrow \infty} P^{n}(G)=1$. Then the following results hold:

(a) $P^{n} \rightarrow Q$ weakly and $S_{Q}=m(S)$ iff $m(S)$ is completely simple with a compact group factor $G$ such that for any proper normal subgroup $H$ of $G, g S_{P} e \cap(G-H) \neq$ $\varnothing$ for each $g \in G-H$, where $e$ is the identity of $G$.

(b) If the rank of the matrices in $m(S)$ is one and $m(S)$ does not contain a subgroup with exactly two elements, then $P^{n}$ converges weakly iff $m(S)$ is completely simple with a compact group factor.

(c) If for each $x \in S$, there is a power $x^{m}$ lying in a subgroup of $S$, then either $P^{n} \rightarrow 0$ vaguely or $\left(P^{n}\right)$ is tight.

ACKNowledgment. The author is grateful to G. Högnäs and P. Bougerol for relevant comments on an earlier version of this paper. The paper [B], using different methods, addresses problems similar to ours under different conditions.

\section{REFERENCES}

[B] Philippe Bougerol, Tightness of products of random matrices and stability of linear stochastic systems, Ann. Probab. 15 (1987), 40-74.

[C] W. Edwin Clark, Remark on the kernel of a matrix semigroup, Czechoslovak Math. J. 15(90) (1965), 305-309.

[HM] G. Högnäs and A. Mukherjea, Recurrent random walks and invariant measures on semigroups of $n \times n$ matrices, Math. Z. 173 (1980), 69-94. 
[KS] Harry Kesten and Frank Spitzer, Convergence in distribution for products of random matrices, Z. Wahrsch. Verw. Gebiete 67 (1984), 363-386.

[M] A. Mukherjea, Limit theorems: Stochastic matrices, ergodic Markov chains and measures on semigroups, Prob. Analysis and Related Topics, Vol. 2, Academic Press, 1979, pp. 143-203.

$\left[\mathbf{M}_{1}\right] \_, A$ new result on the convergence of nonhomogeneous stochastic chains, Trans. Amer. Math. Soc. 262 (1980), 505-520.

[MT] A. Mukherjea and N. A. Tserpes, Measures on topological semigroups, Lecture Notes in Math., vol. 547, Springer-Verlag, Berlin and New York, 1976.

Department of MAThematics, UNiversity of Florida, TAMPA, Florida 33620 